\title{
Corticofugal Modulation of Initial Sound Processing in the Brain
}

\author{
Feng Luo, Qianzhou Wang, Alireza Kashani, and Jun Yan \\ Department of Physiology and Biophysics, Hotchkiss Brain Institute, Faculty of Medicine, University of Calgary, Calgary, Alberta, Canada T2N 4N1
}

The brain selectively extracts the most relevant information in top-down processing manner. Does the corticofugal system, a "back projection system," constitute the neural basis of such top-down selection? Here, we show how focal activation of the auditory cortex with $500 \mathrm{nA}$ electrical pulses influences the auditory information processing in the cochlear nucleus $(\mathrm{CN})$ that receives almost unprocessed information directly from the ear. We found that cortical activation increased the response magnitudes and shortened response latencies of physiologically matched $\mathrm{CN}$ neurons, whereas decreased response magnitudes and lengthened response latencies of unmatched CN neurons. In addition, cortical activation shifted the frequency tunings of unmatched $\mathrm{CN}$ neurons toward those of the activated cortical neurons. Our data suggest that cortical activation selectively enhances the neural processing of particular auditory information and attenuates others at the first processing level in the brain based on sound frequencies encoded in the auditory cortex. The auditory cortex apparently implements a long-range feedback mechanism to select or filter incoming signals from the ear.

Key words: auditory cortex; corticofugal; cochlear nucleus; neural plasticity; neurophysiology; mouse

\section{Introduction}

Hearing in nature encounters noisy environment (Feng and Ratnam, 2000). Behavioral studies from the 1950s have described the brain's automatic selection of sound; one is able to selectively listen to one sound and reject or attenuate the perception of others (Broadbent, 1952; Cherry, 1953). The selection comprises top-down processing based on intention, experience and expectation (Grossberg, 1999; Alain, 2007; Gilbert, 2007). A fundamental issue that lies underneath the automatic sound selection is selective neural processing of auditory information. From the viewpoint of neurobiology, the crucial question is how the brain implements top-down selection. The behavioral characteristics of automatic sound selection determine that the underlying neural substrate must be a back projection system and is able to implement selective sound processing based on auditory information already registered in the higher auditory processing levels.

The corticofugal system is a back projection system capable of providing top-down information flow. There are six hierarchical information processing levels in the central auditory system. The highest level is the auditory cortex where the learned or experienced sounds are registered (Edeline et al., 1993; Recanzone et al., 1993; Bakin and Weinberger, 1996;

\footnotetext{
Received Aug. 20, 2008; revised Sept. 29, 2008; accepted 0ct. 1, 2008.

This work was supported by the Natural Science and Engineering Research Council of Canada, the Hearing Foundation of Canada, the Campbell McLaurin Chair for Hearing Deficiencies of the University of Calgary, and the Alberta Heritage Foundation for Medical Research. We thank Drs. Jos J. Eggermont, Douglas Zochodne, and Robert J. McDonald for their helpful comments on the previous version of this manuscript.

Correspondence should be addressed to Dr. Jun Yan, Department of Physiology and Biophysics, Hotchkiss Brain Institute, Faculty of Medicine, University of Calgary, 3330 Hospital Drive, N.W., Calgary, Alberta, Canada T2N 4N1. E-mail: juyan@ucalgary.ca.

DOl:10.1523/JNEUROSCI.3972-08.2008

Copyright $\odot 2008$ Society for Neuroscience $\quad$ 0270-6474/08/2811615-07\$15.00/0
}

Pantev et al., 1998; Zhang et al., 2001, 2006; Ma and Suga, 2003; Yan and Zhang, 2005; Polley et al., 2006; Alain et al., 2007; Froemke et al., 2007; Ji and Suga, 2007). The lowest level is the cochlear nucleus $(\mathrm{CN})$ that gates all incoming signals from the ear to the brain (Kiang et al., 1967; Evans, 1975; Liberman and Kiang, 1978; Feng and Vater, 1985; Taberner and Liberman, 2005). Neurons in the deep layers of the auditory cortex send descending (corticofugal) fibers directly to most subcortical nuclei including the CN (Winer et al., 2001; Doucet et al., 2002; Coomes and Schofield, 2004a; Schofield and Coomes, 2005; Meltzer and Ryugo, 2006; Bajo et al., 2007; Coomes Peterson and Schofield, 2007). The characteristics of the anatomical structures suggest that the central auditory system has the neural circuitry through which the auditory cortex could implement long-range feedback to control the incoming information from the ear.

Many studies in the visual, auditory and somatosensory systems have unveiled that sensory cortices differentially modulate subcortical neuron activities in a highly specific manner (Sillito et al., 1994; Yan and Suga, 1996; Zhang et al., 1997; Zhou and Jen, 2000, 2007; Yan and Ehret, 2002; Malmierca and Nuñez, 2004; Yan et al., 2005; Castellanos et al., 2007; Ma and Suga, 2007). In the auditory system, focal cortical activation enhances the thalamic and midbrain processing of auditory information that is encoded by activated cortical neurons (Suga and Ma, 2003). Cortical activation even modulates cochlear hair cells in a frequency-specific way (Xiao and Suga, 2002). Because the $\mathrm{CN}$ is the gate of the central auditory system, selective corticofugal modulation of $\mathrm{CN}$ neurons could be an important mechanism for controlling information flow to the brain, i.e., for early sound selection. Up to date, little is known how the auditory cortex improves or adjusts sound information processing in the $\mathrm{CN}$. 


\section{Materials and Methods}

The impact of the auditory cortex on the auditory information processing in the $\mathrm{CN}$ was examined by observing the changes of auditory responses and frequency tunings of $\mathrm{CN}$ neurons after focal electrical stimulation (ES) of the primary auditory cortex (Fig. $1 \mathrm{~A}$ ). Forty-one female C57 mice (Charles River Laboratories) aged 4-7 weeks and weighing 14.6-24.0 g were used in this study. Animal preparation, acoustic stimulation, electrical stimulation of the auditory cortex, recording of action potentials and data processing were mostly the same as those described previously (Yan and Ehret, 2002; Yan et al., 2005; Jafari et al., 2007; Wu and Yan, 2007). All protocols and procedures were in accordance with the Canadian Council on Animal Care and approved by the Animal Care Committee of the University of Calgary (protocol number: M04044).

Animal preparation. Under anesthesia with a mixture of ketamine $(85 \mathrm{mg} / \mathrm{kg}$, i.p.) and xylazine $(15 \mathrm{mg} / \mathrm{kg}$, i.p.), the mouse's head was fixed in a custom-made head holder by rigidly clamping between the palate and nasal/frontal bones. The mouth bar was adjusted to align the bregma and $\lambda$ points of the skull in one horizontal plane. The scalp was incised along the midline and subcutaneous tissue and muscle were removed to expose the skull. Two holes measuring $2 \mathrm{~mm}$ in diameter were drilled in the skull to expose the right cerebellum above the CN (5.1-5.6 mm posterior to bregma, 2.3-2.8 $\mathrm{mm}$ lateral to the midline) and the left auditory cortex. The right $\mathrm{CN}$ and left auditory cortex were selected because the ascending auditory pathway runs from the $\mathrm{CN}$ to the contralateral cortex. During surgery and all recording sessions, the animal's body temperature was maintained at $37^{\circ} \mathrm{C}$ by a feedback-controlled heating pad. Anesthetic levels were maintained by additional doses of ketamine $(17 \mathrm{mg} / \mathrm{kg}$, i.p. $)$ and xylazine $(3 \mathrm{mg} / \mathrm{kg}$, i.p.) when the animal exhibited responses to tail pinching. All experiments were performed in a soundproof chamber.

Acoustic stimulation. Tone bursts $(60 \mathrm{~ms}$ duration with $5 \mathrm{~ms}$ rise and fall times) were used as acoustic stimuli. They were digitally synthesized and then converted into analog sinusoidal waves by an Enhanced Realtime Processor (RP2; Tucker-Davis Technologies). The signals were then fed to a tweeter via a digital attenuator (PA5) and power amplifier. The output amplitude of the tone bursts was expressed as decibel sound pressure level ( $\mathrm{dB}$ SPL, ref. $20 \mu \mathrm{Pa}$ ). The tweeter was placed $45^{\circ}$ to the right of and $130 \mathrm{~cm}$ away from the mouse's right ear. During calibration, the tweeter was driven by 20 volt sinusoidal peak-to-peak bursts without attenuation. The amplitudes of tone bursts were measured and calibrated at the position of animal right ear with a condenser microphone (Model 2520; Larson-Davis Laboratories) and a microphone preamplifier (Model 2200C; Larson-Davis Laboratories). Frequencies and intensities of tone bursts were varied either manually or automatically with BrainWare software (Tucker-Davis Technologies).

Recording and electrical stimulation in the primary auditory cortex. A tungsten electrode of $\sim 2 \mathrm{M} \Omega$ impedance was advanced, perpendicularly to the surface of the auditory cortex. The electrode was initially connected to the recording system. Signals recorded with the electrode were fed to a 16-channel preamplifier and were then amplified 10,000 times and filtered with a bandpass of $0.3-10 \mathrm{kHz}$ with a RA16 module (TuckerDavis Technologies). The output signals were observed on the oscilloscope and computer screen. During the electrode penetration, a tone burst with manual alteration of its frequency and amplitude was contin-
ES \& recording

\section{Recording}
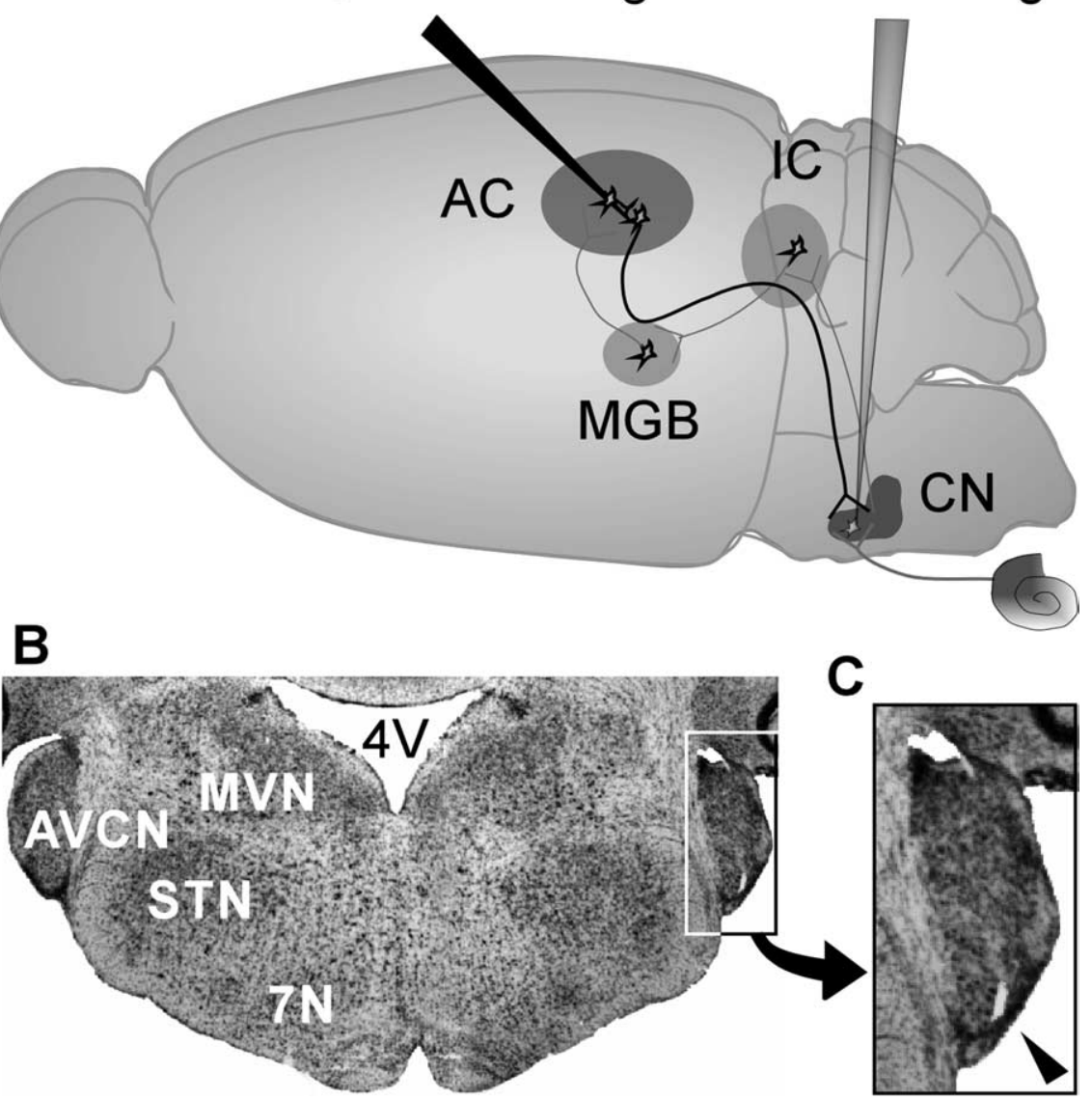

Figure 1. A, A schematic drawing of the mouse brain, including the corticofugal projection to $\mathrm{CN}$ as indicated by a solid dark line, and the sites of electrical stimulation and recording. $\boldsymbol{B}$, Photomicrograph of the coronal section of the right brainstem at $\sim 5.5 \mathrm{~mm}$ posterior to the bregma. $\boldsymbol{C}$, Magnification of the area in a rectangle in $\boldsymbol{B}$. A lesion site can be seen at the anteroventral cochlear nucleus (AVCN) as indicated by an arrowhead. AC, Auditory cortex; IC, inferior colliculus, MGB, medial geniculate body; MVN, medial vestibular nucleus; STN, spinal trigeminal nucleus; $4 \mathrm{~V}$, fourth ventricle; $5 \mathrm{~N}$, facial nucleus.

uously delivered at a rate of 1 per second. Tone-evoked action potentials were frequently found at $\sim 300 \mu \mathrm{m}$ below the brain surface (layers IIIIV) of the auditory cortex. The best frequency (BF) and minimum threshold of the recorded neurons were first measured. The minimum threshold was defined as the lowest sound amplitude across all frequencies and the BF was the frequency to which a neuron showed the largest response magnitude (spike number). The $\mathrm{BF}$ of the recorded neuron was first measured by manual alteration of tone frequency and amplitude. On the average, 5-8 electrode penetrations were made in each animal to determine the location of the primary auditory cortex. The electrode was then placed within the primary auditory cortex and penetrated until tone-evoked action potentials were observed. The BF and minimum threshold of the recorded cortical neurons were measured by manual alteration of tone frequency and amplitude. The responses of cortical neurons to tone bursts at different frequencies $(10 \mathrm{~dB}$ above the minimum threshold) were then recorded and saved with BrainWare software. The BFs of the stimulated cortical neurons were determined based on the saved data. Thereafter, the electrode served as a stimulating electrode by switching the connection from the recording system to the output of an A360 constant current isolator (World Precision Instruments) of the stimulating system. The electrode was further advanced to a depth of $\sim 700-800 \mu \mathrm{m}$ below the brain surface (deep layers) and was maintained in the same position throughout the experiment. The neurons at $700-$ $800 \mu \mathrm{m}$ below the brain surface should have the same BFs as those at 300 $\mu \mathrm{m}$ because of the columnar organization found in the auditory cortex (Abeles and Goldstein, 1970; Suga and Manabe, 1982). Negative pulses (monophasic, $0.1 \mathrm{~ms}, 500 \mathrm{nA}$ constant current), generated by a Grass S88 
A
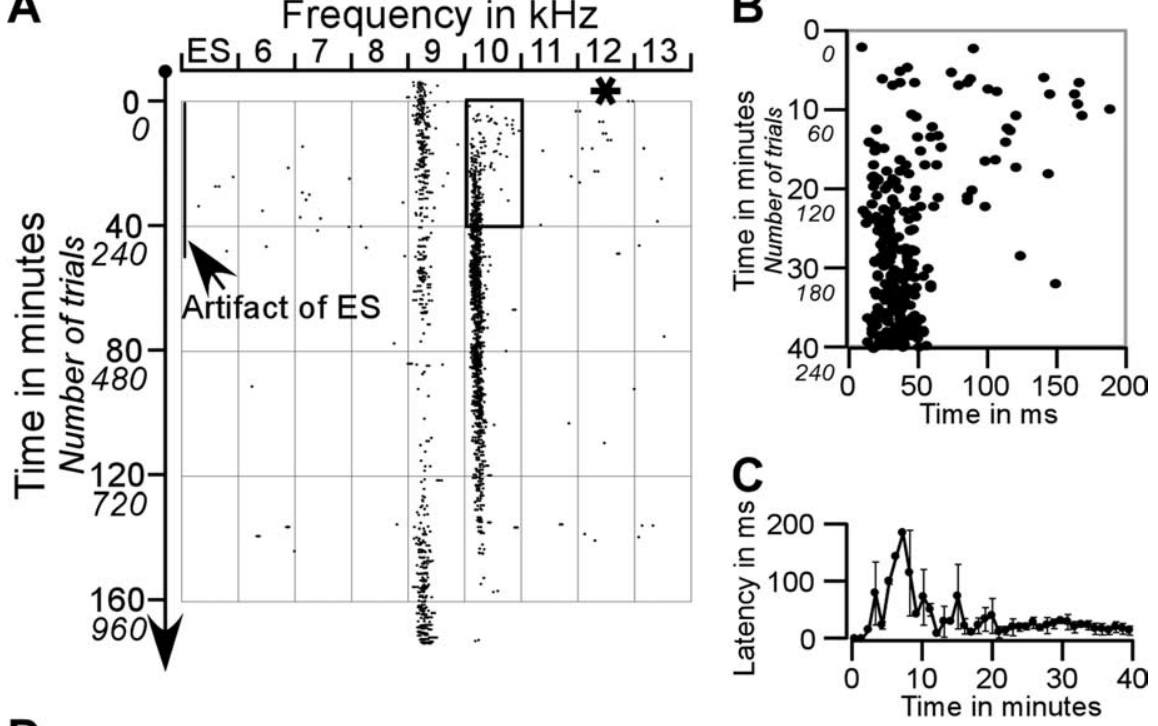

D

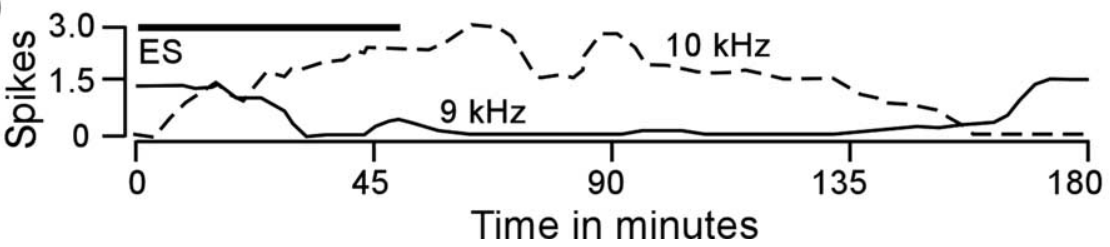

Figure 2. A, Raster plotting of a typical change in auditory responses of a CN neuron before, during, and after ES. B, Magnification of the gray box in $A$, the initial development of the neuronal responses of this neuron to $10 \mathrm{kHz}$ tone during $\mathrm{ES}$. C, The change in the average latency is quantitatively illustrated. $\boldsymbol{D}$. The time courses of changes in response magnitudes of this neuron to both 9 and $10 \mathrm{kHz}$ tones. The asterisk indicates the best frequency of the stimulated cortical neurons.

stimulator (Astro-Medical) and the A360 constant-current unit (World Precision Instrument), were delivered to the primary auditory cortex through this electrode. An indifferent electrode for recording and electrical stimulation was placed on the brain surface just beside the stimulating electrode.

Recording in the $C N$. Two tungsten electrodes of $\sim 2 \mathrm{M} \Omega$ impedance separated by $100 \mu \mathrm{m}$ were connected to the TDT 16-channel preamplifier of the recording system and dorsoventrally advanced into the CN. During the electrode penetration, a tone burst with manual alteration of its frequency and amplitude was continuously delivered at a rate of 1 per second. Tone-evoked action potentials were commonly observed when the electrode tips were $2.5-3.6 \mathrm{~mm}$ below the surface of the cerebellum. The electrodes were then removed. Another penetration was made $\sim 100 \mu \mathrm{m}$ anterior to the previous penetration and the same procedure continued until no tone-evoked action potentials were observed. Then the penetration was made $100 \mu \mathrm{m}$ either lateral or medial to previous penetrations and the above procedure repeated until the rostral boundary of the $\mathrm{CN}$ was determined. The electrodes were again removed. A final penetration was made in a range of $25-300 \mu \mathrm{m}$ caudal to the rostral boundary of the $\mathrm{CN}$ for recording the auditory responses of neurons in the anteroventral division of the $\mathrm{CN}$. Once tone-evoked action potentials of $\mathrm{CN}$ neurons were observed, the $\mathrm{BF}$ and minimum threshold were audiovisually measured with various tone bursts delivered at a rate of 4 per second. The responses of $\mathrm{CN}$ neurons to various tone bursts were then recorded and saved with Brainware software (Tucker-Davis Technologies).

Experimental protocols. Once the BFs and minimum thresholds of $\mathrm{CN}$ neurons were measured, two experimental protocols were applied to evaluate the influence of the cortex on the activities of $\mathrm{CN}$ neurons.

One emphasized or highlighted how fast the changes were in $\mathrm{CN}$ auditory responses and how the response latency of the $\mathrm{CN}$ neuron was affected after cortical stimulation. In this protocol, a series of tone bursts separated by $500 \mathrm{~ms}$ was delivered to obtain frequency-dependent re- sponses of recorded $\mathrm{CN}$ neurons. The tone frequency varied from $5 \mathrm{kHz}$ below to $5 \mathrm{kHz}$ above the $\mathrm{BFs}$ of given $\mathrm{CN}$ neurons with a $1 \mathrm{kHz}$ increment. The tone amplitude was set at the minimum thresholds of given $\mathrm{CN}$ neurons so that neurons could only show responses to a few tone frequencies before cortical stimulation. A series of tone bursts was delivered every $10 \mathrm{~s}$. The responses of $\mathrm{CN}$ neurons to the first 50 trials were recorded as control data. Starting from the 51st trial, a single electrical pulse was delivered to the primary auditory cortex $500 \mathrm{~ms}$ before the onset of the first tone burst. After 250 trials, the ES stopped but the tone bursts were continuously delivered until the ES-evoked changes in the auditory responses of $\mathrm{CN}$ neurons were recovered.

The second protocol focused on the frequency-dependent changes in $\mathrm{CN}$ auditory responses after cortical stimulation. Because this protocol was identical to what was used in our previous studies (Yan and Ehret, 2002; Yan et al., 2005), data were comparable with our previous findings. This protocol sampled the frequency tunings of $\mathrm{CN}$ neurons with a series of tone bursts separated by $250 \mathrm{~ms}$. Tone frequency at $10 \mathrm{~dB}$ above the minimum thresholds of $\mathrm{CN}$ neurons varied from 3 to $40 \mathrm{kHz}$ with a $1.0 \mathrm{kHz}$ increment. The identical tone stimulation was repeated 15 times. After sampling the control data, electrical pulses were delivered to the primary auditory cortex at a rate of 4 per second for $7 \mathrm{~min}$. Immediately after ES, the frequency tuning of the recorded $\mathrm{CN}$ neuron was continuously sampled with the same tone set every 30 min until the ES-evoked changes in frequency tuning were disappeared.

Data processing. Single-unit action potentials were isolated from the multiunit recording according to eight parameters of the waveform of action potentials; these parameters were peak, valley, spike height, spike width, peak time, valley time and two user-defined voltage values. The evaluated data included only neurons with stable spike waveforms over the entire recording session. Single-unit responses to a series of tone bursts were eventually displayed by dot rasters or peristimulus time histograms with a bin width of $1 \mathrm{~ms}$.

In protocol one, the response latency, first-spike latency, and spike numbers were calculated based on 1 min time window (six-tone stimuli). The response latency of $\mathrm{CN}$ neurons was the average latency of all spikes, the first spike latency was the average latency of all first spikes and spike number was the count of spikes within the time window.

In protocol two, the excitatory frequency-response curve was derived from the response magnitudes of a neuron to a series of tone bursts.

Histology. On completion of the recording session, a 20-s-long and $1 \mathrm{~mA}$ electrical current was applied to the recording site in the $\mathrm{CN}$ through the recording and indifferent electrodes. The animal was given a cardiac perfusion with $10 \mathrm{ml}$ of physiological saline and a mixture of $4 \%$ paraformaldehyde in $0.1 \mathrm{~m}$ phosphate buffer of $\mathrm{pH} 7.4$. The brain was then removed from the cranium, fixed by immersion in $4 \%$ paraformaldehyde in a $0.1 \mathrm{~m}$ phosphate buffer and stored in phosphate buffered saline solution containing $20 \%$ sucrose at $4^{\circ} \mathrm{C}$. After the brain was embedded with OCT (optimal cutting temperature) compound and frozen in 2-methylbutane, $40 \mu \mathrm{m}$ coronal brainstem sections were made with a cryostat. The tissue sections were mounted onto glass slides and stained using the Nissl method. The electrolytic lesion was examined under a light microscope. An example is shown in Figure 1, $B$ and $C$.

Statistics. Data were expressed as mean $\pm \mathrm{SD}$. A $t$ test was used to compare the differences between groups of data and numbers. A $p$ value of $<0.05$ was considered to be statistically significant. 


\section{Results}

We first examined the changes in response properties of $\mathrm{CN}$ neurons after focal ES of the auditory cortex in mice. Figure 2 shows an example of changes in responses of a $\mathrm{CN}$ neuron before, during and after the ES. This CN neuron originally showed strong response to a $9 \mathrm{kHz}$ tone burst but no response to other tones including a $10 \mathrm{kHz}$ tone burst (Fig. $2 \mathrm{~A}$, dots above zero). When $12-\mathrm{kHz}$-tuned cortical neurons were electrically stimulated, however, the response of this $\mathrm{CN}$ neuron to a $9 \mathrm{kHz}$ tone burst gradually decreased and finally ceased. In contrast, its response to a $10 \mathrm{kHz}$ tone burst emerged (Fig. $2 \mathrm{~A}$ ). The buildup of the response to the $10 \mathrm{kHz}$ tone burst was closely observed. This $\mathrm{CN}$ neuron exhibited its first spike in response to the $10 \mathrm{kHz}$ tone burst at the 12th cortical stimulus (120 s after the first ES). From the 12th stimulus to the 120th stimulus (first $20 \mathrm{~min}$ of ES), the number of spikes of this neuron in response to the $10 \mathrm{kHz}$ tone burst gradually increased with extremely long spike latencies. After the 120th stimulus (20 min), this neuron showed much stronger response with greatly reduced response spike latencies (Fig. $2 B)$. The changes in response latencies are quantified in Figure $2 C$ in which the average latency of all spikes in a $1 \mathrm{~min}$ (six cortical stimuli) time window was calculated. The change in auditory response of this neuron slowly disappeared after the cessation of ES as shown in Figure $1 D$ in which the average spike numbers in $1 \mathrm{~min}$ (six cortical stimuli) time windows were calculated. At $20 \mathrm{~min}$ after ES (120 cortical stimuli), this neuron showed equal response magnitudes to $9 \mathrm{kHz}$ and $10 \mathrm{kHz}$. From this time point, the spike number in response to $10 \mathrm{kHz}$ exceeded that to $9 \mathrm{kHz}$. As a result, the $\mathrm{BF}$ of this $\mathrm{CN}$ neuron shifted from $9 \mathrm{kHz}$ to $10 \mathrm{kHz}$, i.e., toward the $12 \mathrm{kHz}$, the BF of the stimulated cortical neurons. The response to $9 \mathrm{kHz}$ tended to cease at $31 \mathrm{~min}$ after the onset of ES (186 cortical stimuli). The responses of this neuron to $9 \mathrm{kHz}$ and $10 \mathrm{kHz}$ recovered at $158 \mathrm{~min}$ after the onset of ES (108 min after the cessation of ES).

Using the same procedure, we studied seven $\mathrm{CN}$ neurons in which the BFs were $<1 \mathrm{kHz}$ away from those of stimulated cortical neurons and another seven $\mathrm{CN}$ neurons in which the BFs were $>1 \mathrm{kHz}$ away from those of the stimulated cortical neurons. For simplicity, we called the former physiologically matched neurons and the latter physiologically unmatched neurons. Without exception, ES facilitated the auditory responses of matched CN neurons and suppressed those of unmatched $\mathrm{CN}$ neurons. In addition, the BFs of the unmatched neurons shifted toward the BFs of the stimulated cortical neurons (Fig. 2). Such BF-related changes in auditory response magnitudes and BFs of $\mathrm{CN}$ neurons will be addressed later with a procedure used in our previous studies (Yan and Ehret, 2002; Yan et al., 2005).

The data presented in Figure 2 also shows two additional phenomena. One is that the response latency increased at $9 \mathrm{kHz}$ but decreased at $10 \mathrm{kHz}$ after ES (Fig. $2 \mathrm{~A}$ ). The other is that the latency of ES-evoked changes in response magnitude was clearly shorter to the $10 \mathrm{kHz}$ than to $9 \mathrm{kHz}$ (Fig. 2D). This confirms that ES-evoked facilitation occurred more rapidly and was accompanied with a reduced response latency, whereas ES-evoked inhibition occurred at a later period, accompanied by an increased response latency. It was uncertain whether or not these two phenomena were applicable to the ES-evoked facilitation of the auditory responses of matched neurons and inhibition of unmatched neurons. To clarify this issue, the first-spike latencies of the recorded $\mathrm{CN}$ neurons were calculated in $1 \mathrm{~min}$ (six stimuli) time windows before, during and after the ES. Our data demonstrated that the averaged first-spike latency of seven matched

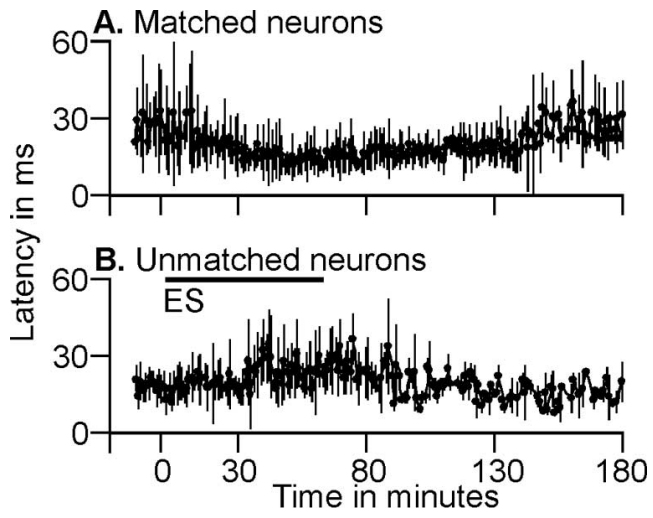

Figure 3. $\boldsymbol{A}, \boldsymbol{B}$, Changes in first spike latencies of matched $(\boldsymbol{A})$ and unmatched $(\boldsymbol{B}) \mathrm{CN}$ neurons as the function of time from the onset of ES.

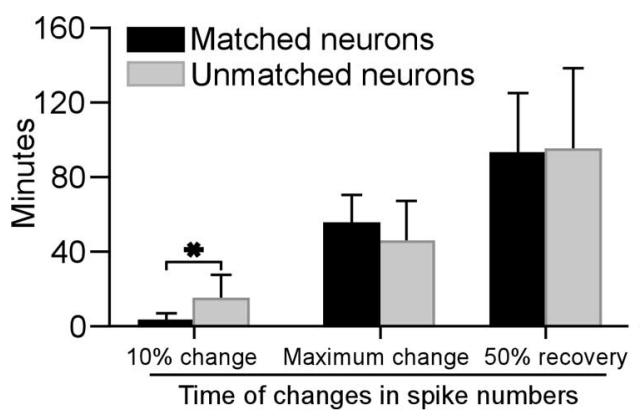

Figure 4. Time required for $10 \%$ change, maximum changes, and $50 \%$ recovery in response magnitudes of the matched and unmatched neurons after ES. ${ }^{*} p<0.05$, when matched and unmatched neurons were compared.

neurons gradually decreased (Fig. $3 A$ ) and that of seven unmatched neurons gradually increased (Fig. $3 B$ ) until the cessation of the ES.

We next calculated the periods of time required for a $10 \%$ change, the maximum change and a 50\% recovery in the response magnitudes of CN neurons after the ES (Fig. 4). The time required for a $10 \%$ increase in the auditory response magnitudes of matched neurons was $2.6 \pm 3.8 \mathrm{~min}(n=7)$, which was significantly shorter than $14.6 \pm 12.8 \mathrm{~min}(n=7)$, the time required for a $10 \%$ decrease in response magnitudes of unmatched neurons $(p<0.05)$. The times of maximum change and 50\% recovery were $54.7 \pm 14.7 \mathrm{~min}$ and $93.0 \pm 32.7 \mathrm{~min}(n=7)$ for matched neurons, which were similar to $45.3 \pm 22.9 \mathrm{~min}$ and $94.7 \pm 43.0$ $\min (n=7)$ for unmatched neurons $(p>0.05)$. The ES influence occurred significantly earlier on matched $\mathrm{CN}$ neurons than that on unmatched ones.

To obtain comparable data regarding the frequency-specific changes in auditory response magnitudes and BFs of $\mathrm{CN}$ neurons, we measured frequency response curves (receptive fields) of $55 \mathrm{CN}$ neurons before and after the ES by using our second protocol.

Three examples in Figure 5 show how ES changed the frequency tunings of $\mathrm{CN}$ neurons. In Figure $5 A$, the BFs of both cortical and $\mathrm{CN}$ neurons were $15 \mathrm{kHz}$. The ES augmented the auditory responses but did not alter the $\mathrm{BF}$ of this $\mathrm{CN}$ neuron. Figure $5 B$ shows that the $\mathrm{BF}$ of this $\mathrm{CN}$ neuron shifted from 17 to $19 \mathrm{kHz}$ after $22-\mathrm{kHz}$-tuned cortical neurons were stimulated, which resulted from the inhibition of the responses to tone bursts $<18 \mathrm{kHz}$ and the facilitation of the responses to tone bursts $>18$ $\mathrm{kHz}$ (Fig. 5B3). The overall response magnitude of this neuron 

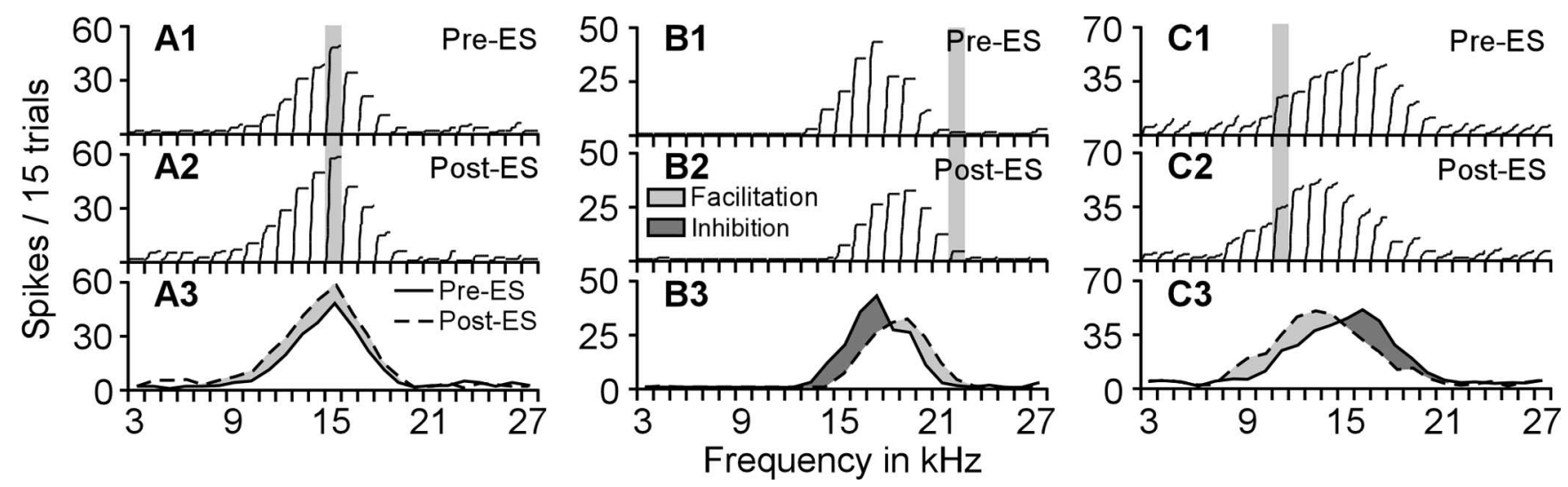

Figure 5. Three examples of the ES effects on frequency tunings of CN neurons. $A-C$, The ES did not change the BF but augmented the auditory responses of the matched $C N$ neurons $(A)$, whereas the ES shifted the BFs of the unmatched CN neurons toward the cortical BF $(\boldsymbol{B}, \boldsymbol{C})$. The shift of BFs resulted from differential facilitation and inhibition (B3, $\mathbf{C})$. The gray bars in the top two panels represent the BFs of the stimulated cortical neurons. In the bottom, the dark gray area represents inhibition, whereas the light gray area represents facilitation.

was suppressed; the spike number in the peak response to $17 \mathrm{kHz}$ tone bursts before the ES was larger than that to $19 \mathrm{kHz}$ tone bursts after the ES. In contrast, the neuron in Figure $5 C$ shifted its BF from $16 \mathrm{kHz}$ down to $13 \mathrm{kHz}$ after an 11-kHz-tuned cortical neurons were stimulated, which resulted from the inhibition of responses to tone bursts $>15 \mathrm{kHz}$ and the facilitation of responses to tone bursts $<15 \mathrm{kHz}$ (Fig. 5C3). These examples clearly demonstrate that the ES could lead to completely different changes in auditory response magnitudes and frequency tunings of $\mathrm{CN}$ neurons, i.e., either the facilitation or inhibition as well as $\mathrm{BF}$ changes in either direction that were largely dependent on the BFs of the stimulated cortical neurons.

Analysis of all 55 neurons confirmed that the ES-evoked changes in response magnitudes of $\mathrm{CN}$ neurons were closely related to the BF differences between the stimulated cortical and the recorded $\mathrm{CN}$ neurons. After the ES, the auditory response magnitudes of $\mathrm{CN}$ neurons increased when the BF differences between the cortical and $\mathrm{CN}$ neurons were $<1.0 \mathrm{kHz}$ (matched neurons), whereas they decreased when the BF differences between the cortical and $\mathrm{CN}$ neurons were larger than $1.0 \mathrm{kHz}$ (unmatched neurons). On the average, the response magnitude of matched neurons increased by $2.2 \pm 10.1 \%$ and those of unmatched neurons decreased by $17.9 \pm 14.5 \%$ when $\mathrm{CN}$ BFs were higher than cortical $\mathrm{BF}$ and by $19.8 \pm 15.1 \%$ when $\mathrm{CN}$ BFs were lower than cortical BF (Fig. 6A). The ES-evoked changes in response magnitude were significantly different between matched and unmatched $\mathrm{CN}$ neurons $(p<0.001)$. The ES-evoked BF shifts of $\mathrm{CN}$ neurons were also correlated to the stimulated cortical BFs (Fig. 6B). For unmatched CN neurons, ES shifted CN BFs either higher or lower depending on the BFs of the stimulated cortical neurons. This was particularly the case when the BF differences between cortical and $\mathrm{CN}$ neurons ranged from -5 to 7 $\mathrm{kHz}$. In other words, ES shifted CN BFs toward the BFs of the stimulated cortical neurons. However, ES did not alter the BFs of matched CN neurons.

\section{Discussion}

Our data clearly suggest that the auditory cortex specifically modulates the neural processing of auditory information in the cochlear nucleus in two ways. One is that cortical stimulation increased the auditory responses and shortened response latencies of the matched $\mathrm{CN}$ neurons but decreased and lengthened those of the unmatched neurons. The second involves the enhancement of the $\mathrm{CN}$ representation of the sound frequencies that are
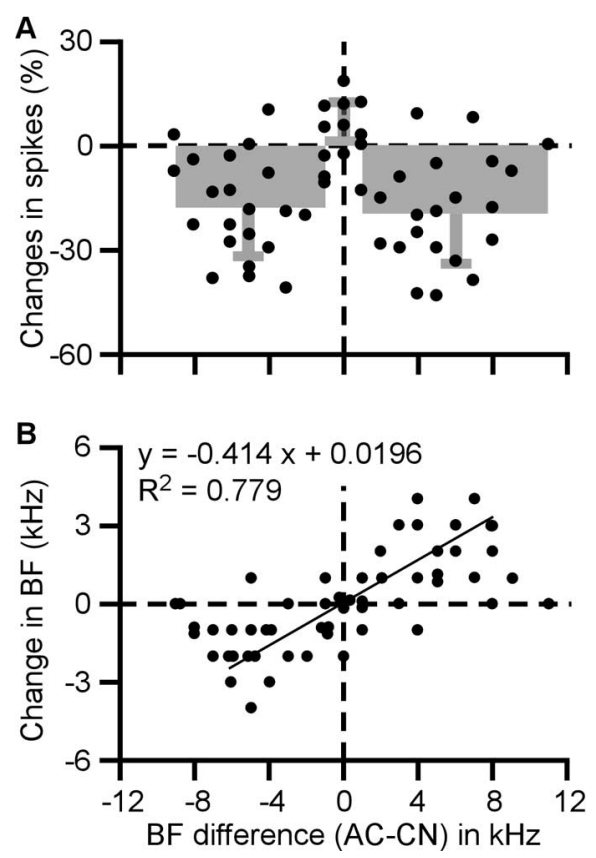

Figure 6. $\boldsymbol{A}, \boldsymbol{B}, \mathrm{ES}$-evoked changes in response magnitudes $(\boldsymbol{A})$ and $\mathrm{BFs}(\boldsymbol{B})$ of $\mathrm{CN}$ neurons as the function of $B F$ difference between the stimulated cortical and recorded $C N$ neurons. These changes were clearly different between the matched and unmatched $\mathrm{CN}$ neurons. The gray boxes and bars in $A$ represent the mean $\pm S D$ of $B F$ shifts of all neurons in its representing range of $B F$ difference, and the line in $\boldsymbol{B}$ is regression line.

emphasized in the auditory cortex, i.e., the shift of the BFs of unmatched $\mathrm{CN}$ neurons toward those of the activated cortical neurons. These findings support a recent report that musical experience enhanced human brainstem encoding of linguistic pitch presumably via corticofugal feedback (Wong et al., 2007). Such corticofugal modulation is consistent with our previous work involving the mouse midbrain. The mouse study reveals that focal cortical activation suppresses auditory responses and shifts $\mathrm{BFs}$ of unmatched midbrain neurons toward the BFs of the activated cortical neurons (Yan and Ehret, 2002; Yan et al., 2005). An important distinction here is that, for unmatched neurons, the ES suppresses auditory responses by up to $80 \%$ in the midbrain comparing with up to $43 \%$ in the CN (Fig. 6A). Similarly, the ratio of ES-evoked $\mathrm{BF}$ shift over $\mathrm{BF}$ difference is 0.58 in the midbrain but 0.41 in the $\mathrm{CN}$ (Fig. 6 B). Earlier bat studies have already 
demonstrated that the ES-evoked changes in auditory responses and BFs are larger in the auditory thalamus than those in the midbrain (Zhang et al., 1997; Zhang and Suga, 2000). Interestingly, the auditory cortex also implements highly specific modulation of auditory receptors in the cochlea via olivocochlear neurons; such modulation is probably smaller than corticofugal modulation of central nuclei (Xiao and Suga, 2002; Perrot et al., 2006). This suggests that corticofugal modulation cascades from the auditory thalamus down to the auditory periphery.

With all of these findings considered, a framework of a physiological model of top-down selection emerges in the central auditory system. The auditory cortex is an adaptive processor of auditory experience and learning (Dahmen and King, 2007). Because of the impact of top-down modulation through the corticofugal system, the auditory cortex automatically selects incoming signals from the ear based on cortical information that is emphasized through previous auditory learning and experience. The incoming signals are amplified if they match the emphasized information in the auditory cortex. Conversely, the incoming signals are attenuated or suppressed if they are not or are relatively less emphasized (Fig. 6). The differential changes in various information (frequency) channels are likely based on cortical impact on dynamics of auditory nerve-to-CN synaptic transmission and/or inhibitory modulation of $\mathrm{CN}$ neurons (Figs. $2 A, B, 3 A, 5$ ). This top-down selection enhances step by step through corticofugal projections to other subcortical nuclei along the ascending auditory system. It should be noted here that cortical neurons also send descending fibers to the inferior colliculus and superior olivary complex that in turn project to the $\mathrm{CN}$ (Horváth et al., 2000; Doucet et al., 2002; Coomes and Schofield, 2004a,b; Bajo et al., 2007; Coomes Peterson et al., 2007). Therefore, the ES-evoked $\mathrm{CN}$ changes observed in this study can be a summation of direct and indirect corticofugal influences.

There are three fundamental requirements for the selective sound processing for hearing in nature. First of all, selective processing is directed by higher-order information processes. This requires a top-down mechanism that compares the registered information with incoming signals. Only the matched signal is selected and amplified, whereas others are suppressed (Cherry, 1953; Grossberg, 1999; Ahveninen et al., 2006; Gilbert and Sigman, 2007; Knudsen, 2007). Second, the higher-order processing levels should have efficient feedback pathways to lower-order processing levels. Because the top-down mechanism was originally proposed for the impact of higher-order cortical areas to lower-order cortical areas, the focus has been primarily on the intracortical feedback pathway. Recent evidence suggests that the impact of the cortex on subcortical nuclei must also be taken into account (Grossberg, 1999; Alain, 2007). Last, selective processing should ideally take place as early as possible so that the brain receives less irrelevant sound information and neural selective processing can be more efficient. Our work allows us to propose with considerable certainty that the corticofugal system, the topdown selection, is an effective neural substrate that comprises these elements or satisfies these requirements of automatic sound selection in the brain for hearing in nature.

\section{References}

Abeles M, Goldstein MH Jr (1970) Functional architecture in cat primary auditory cortex: columnar organization and organization according to depth. J Neurophysiol 33:172-187.

Ahveninen J, Jääskeläinen IP, Raij T, Bonmassar G, Devore S, Hämäläinen M, Levänen S, Lin FH, Sams M, Shinn-Cunningham BG, Witzel T, Belliveau JW (2006) Task-modulated "what" and "where" pathways in human auditory cortex. Proc Natl Acad Sci U S A 103:14608-14613.
Alain C (2007) Breaking the wave: effects of attention and learning on concurrent sound perception. Hear Res 229:225-236.

Alain C, Snyder JS, He Y, Reinke KS (2007) Changes in auditory cortex parallel rapid perceptual learning. Cereb Cortex 17:1074-1084.

Bajo VM, Nodal FR, Bizley JK, Moore DR, King AJ (2007) The ferret auditory cortex: descending projections to the inferior colliculus. Cereb Cortex 17:475-491.

Bakin JS, Weinberger NM (1996) Induction of a physiological memory in the cerebral cortex by stimulation of the nucleus basalis. Proc Natl Acad Sci U S A 93:11219-11224.

Broadbent DE (1952) Listening to one of two synchronous messages. J Exp Psychol 44:51-55.

Castellanos NP, Malmierca E, Nuñez A, Makarov VA (2007) Corticofugal modulation of the tactile response coherence of projecting neurons in the gracilis nucleus. J Neurophysiol 98:2537-2549.

Cherry EC (1953) Some experiments on the recognition of speech, with one and two ears. J Acoust Soc Am 25:975-979.

Coomes DL, Schofield BR (2004a) Projections from the auditory cortex to the superior olivary complex in guinea pigs. Eur J Neurosci 19:2188-2200.

Coomes DL, Schofield BR (2004b) Separate projections from the inferior colliculus to the cochlear nucleus and thalamus in guinea pigs. Hear Res 191:67-78.

Coomes Peterson D, Schofield BR (2007) Projections from auditory cortex contact ascending pathways that originate in the superior olive and inferior colliculus. Hear Res 232:67-77.

Dahmen JC, King AJ (2007) Learning to hear: plasticity of auditory cortical processing. Curr Opin Neurobiol 17:456-464.

Doucet JR, Rose L, Ryugo DK (2002) The cellular origin of corticofugal projections to the superior olivary complex in the rat. Brain Res 925:28-41.

Edeline JM, Pham P, Weinberger NM (1993) Rapid development of learning-induced receptive field plasticity in the auditory cortex. Behav Neurosci 107:539-551.

Evans EF (1975) Cochlear nerve and cochlear nucleus. In: Handbook of sensory physiology (Keidel WD, Neff WD, eds), pp 1-108. Berlin: Springer.

Feng AS, Ratnam R (2000) Neural basis of hearing in real-world situations. Annu Rev Psychol 51:699-725.

Feng AS, Vater M (1985) Functional organization of the cochlear nucleus of rufous horseshoe bats (Rhinolophus rouxi): frequencies and internal connections are arranged in slabs. J Comp Neurol 235:529-553.

Froemke RC, Merzenich MM, Schreiner CE (2007) A synaptic memory trace for cortical receptive field plasticity. Nature 450:425-429.

Gilbert CD, Sigman M (2007) Brain states: top-down influences in sensory processing. Neuron 54:677-696.

Grossberg S (1999) The link between brain learning, attention, and consciousness. Conscious Cogn 8:1-44.

Horváth M, Kraus KS, Illing RB (2000) Olivocochlear neurons sending axon collaterals into the ventral cochlear nucleus of the rat. J Comp Neurol 422:95-105.

Jafari MR, Zhang Y, Yan J (2007) Multiparametric changes in the receptive field of cortical auditory neurons induced by thalamic activation in the mouse. Cereb Cortex 17:71-80.

Ji W, Suga N (2007) Serotonergic modulation of plasticity of the auditory cortex elicited by fear conditioning. J Neurosci 27:4910-4918.

Kiang NY, Sachs MB, Peake WT (1967) Shapes of tuning curves for single auditory-nerve fibers. J Acoust Soc Am 42:1341-1342.

Knudsen EI (2007) Fundamental components of attention. Annu Rev Neurosci 30:57-78.

Liberman MC, Kiang NY (1978) Acoustic trauma in cats: cochlear pathology and auditory-nerve activity. Acta Otolaryngol [Suppl] 358:1-63.

Ma X, Suga N (2003) Augmentation of plasticity of the central auditory system by the basal forebrain and/or somatosensory cortex. J Neurophysiol 89:90-103.

MaX, Suga N (2007) Multiparametric corticofugal modulation of collicular duration-tuned neurons: modulation in the amplitude domain. J Neurophysiol 97:3722-3730.

Malmierca E, Nuñez A (2004) Primary somatosensory cortex modulation of tactile responses in nucleus gracilis cells of rats. Eur J Neurosci 19:1572-1580.

Meltzer NE, Ryugo DK (2006) Projections from auditory cortex to cochlear 
nucleus: A comparative analysis of rat and mouse. Anat Rec A Discov Mol Cell Evol Biol 288:397-408.

Pantev C, Oostenveld R, Engelien A, Ross B, Roberts LE, Hoke M (1998) Increased auditory cortical representation in musicians. Nature 392:811-814.

Perrot X, Ryvlin P, Isnard J, Guénot M, Catenoix H, Fischer C, Mauguière F, Collet L (2006) Evidence for corticofugal modulation of peripheral auditory activity in humans. Cereb Cortex 16:941-948.

Polley DB, Steinberg EE, Merzenich MM (2006) Perceptual learning directs auditory cortical map reorganization through top-down influences. J Neurosci 26:4970-4982.

Recanzone GH, Schreiner CE, Merzenich MM (1993) Plasticity in the frequency representation of primary auditory cortex following discrimination training in adult owl monkeys. J Neurosci 13:87-103.

Schofield BR, Coomes DL (2005) Auditory cortical projections to the cochlear nucleus in guinea pigs. Hear Res 199:89-102.

Sillito AM, Jones HE, Gerstein GL, West DC (1994) Feature-linked synchronization of thalamic relay cell firing induced by feedback from the visual cortex. Nature 369:479-482.

Suga N, Ma X (2003) Multiparametric corticofugal modulation and plasticity in the auditory system. Nat Rev Neurosci 4:783-794.

Suga N, Manabe T (1982) Neural basis of amplitude-spectrum representation in auditory cortex of the mustached bat. J Neurophysiol 47:225-255.

Taberner AM, Liberman MC (2005) Response properties of single auditory nerve fibers in the mouse. J Neurophysiol 93:557-569.

Winer JA, Diehl JJ, Larue DT (2001) Projection from auditory cortex to the medial geniculate body of the cat. J Comp Neurol 430:27-55.

Wong PC, Skoe E, Russo NM, Dees T, Kraus N (2007) Musical experience shapes human brainstem encoding of linguistic pitch patterns. Nat Neurosci 10:420-422.

Wu Y, Yan J (2007) Modulation of the receptive fields of midbrain neurons elicited by thalamic electrical stimulation through corticofugal feedback. J Neurosci 27:10651-10658.

Xiao Z, Suga N (2002) Modulation of cochlear hair cells by the auditory cortex in the mustached bat. Nat Neurosci 5:57-63.

Yan J, Ehret G (2002) Corticofugal modulation of midbrain sound processing in the house mouse. Eur J Neurosci 16:119-128.

Yan J, Suga N (1996) Corticofugal modulation of time-domain processing of biosonar information in bats. Science 273:1100-1103.

Yan J, Zhang Y (2005) Sound-guided shaping of the receptive field in the mouse auditory cortex by basal forebrain activation. Eur J Neurosci 21:563-576.

Yan J, Zhang Y, Ehret G (2005) Corticofugal shaping of frequency tuning curves in the central nucleus of the inferior colliculus of mice. J Neurophysiol 93:71-83.

Zhang LI, Bao S, Merzenich MM (2001) Persistent and specific influences of early acoustic environments on primary auditory cortex. Nat Neurosci 4:1123-1130.

Zhang Y, Suga N (2000) Modulation of responses and frequency tuning of thalamic and collicular neurons by cortical activation in mustached bats. J Neurophysiol 84:325-333.

Zhang Y, Suga N, Yan J (1997) Corticofugal modulation of frequencyprocessing in bats auditory system. Nature 387:900-903.

Zhang Y, Hamilton SE, Nathanson NM, Yan J (2006) Decreased inputspecific plasticity of the auditory cortex in mice lacking M1 muscarinic acetylcholine receptors. Cereb Cortex 16:1258-1265.

Zhou X, Jen PH (2000) Brief and short-term corticofugal modulation of subcortical auditory responses in the big brown bat, Eptesicus fuscus. J Neurophysiol 84:3083-3087.

Zhou X, Jen PH (2007) Corticofugal modulation of multi-parametric auditory selectivity in the midbrain of the big brown bat. J Neurophysiol 98:2509-2516. 